The Astrophysical Journal, 618:1074-1078, 2005 January 10

(C) 2005. The American Astronomical Society. All rights reserved. Printed in U.S.A.

\title{
A TRANSITION TO FAST FLOWS AND ITS EFFECTS ON THE MAGNETIC FIELDS AND COSMIC RAYS OBSERVED BY VOYAGER 2 NEAR 70 AU
}

\author{
L. F. Burlaga, ${ }^{1}$ N. F. Ness, ${ }^{2}$ C. Wang, ${ }^{3,4}$ J. D. Richardson, ${ }^{3}$ F. B. McDonald, ${ }^{5}$ and E. C. Stone ${ }^{6}$ \\ Received 2004 May 31; accepted 2004 September 21
}

\begin{abstract}
A shocklike transition at 2003.37 from quasi-periodic flows to increasingly fast flows was observed by Voyager 2 (V2) near $70 \mathrm{AU}$. The transition was related to the appearance of coronal holes near the solar equatorial plane on Carrington rotation $(C R) \approx 1994$. Several important features (two cycles of quasi-periodic variations in $V$ and $B$, a shock, a merged interaction region (MIR) with strong magnetic fields in a region with increasing speed, and a second shock that was related to the 2003 October 29-30 events at $1 \mathrm{AU}$ ) were observed by $V 2$ from 2002.8 to 2004.4. A one-dimensional multifluid MHD model shows that these features at $V 2$ evolved from the flows observed by $A C E$ at $1 \mathrm{AU}$. The model also shows that the features propagated through the heliosphere out to $95 \mathrm{AU}$. The changes in the magnetic field associated with the features at $V 2$ caused changes in the cosmic-ray intensity (CRI) of particles more energetic than $70 \mathrm{MeV}$ nucleon $^{-1}$. The quasi-periodic variations were related to variations in CRI, but they produced no appreciable net decrease in the CRI. Both the first shock and the MIR produced a steplike decrease in the CRI at $V 2$.
\end{abstract}

Subject headings: cosmic rays - interplanetary medium

\section{INTRODUCTION}

Voyager 2 ( V2) observed an abrupt transition at 2003.37 from quasi-periodic flows with a mean speed $\approx 390 \mathrm{~km} \mathrm{~s}^{-1}$ to increasingly fast flows with speeds approaching $\approx 550 \mathrm{~km} \mathrm{~s}^{-1}$ at 2004.4 (§ 2). From 2002.8 to $2004.4, V 2$ moved from 68.4 to $73.7 \mathrm{AU}$ at latitudes from $23^{\circ} .9$ south to $25^{\circ} .5$ south, respectively. It was suggested that Voyager $1(\mathrm{Vl})$ crossed the termination shock in 2002 near 85 AU (Krimigis et al. 2003), but the magnetic field observations (Burlaga et al. 2003b) and the energetic particle observations of McDonald et al. (2003) indicate that $V 1$ did not cross the termination shock. A plot of the trajectories of $V 1$ and $V 2$ can be found in the paper of Richardson et al. 2004.

The increasing speeds at $V 2$ were related to increasing speeds at $A C E$ at $1 \mathrm{AU}$ and to the appearance and growth of large coronal holes near the equatorial plane. Section 2 shows that the speed profile at $V 2$ is explained by the one-dimensional multifluid MHD of Wang (Wang \& Richardson 2001; Wang et al. 2000 ) for the boundary conditions at $1 \mathrm{AU}$ given by the $A C E$ magnetic field and plasma observations in the ecliptic.

Prior to the shocklike transition at 2003.37, from before 2002.7 to $2003.37, V 2$ observed positively correlated, quasiperiodic variations in the solar wind speed $(V)$ and density $(N)$ and the magnetic field strength $(B)$ (Richardson et al. 2003). Following the shock, there was an increase in $B$. Another increase in $B$ was observed near the end of 2003 in a merged interaction region (MIR) (Burlaga 1995) across which the

\footnotetext{
1 NASA Goddard Space Flight Center, Code 692, Greenbelt, MD 20771; leonard.f.burlaga@nasa.gov.

2 Bartol Research Institute, University of Delaware, Newark, DE 19716; nfness@udel.edu.

3 Center for Space Research, Massachusetts Institute of Technology, Cambridge, MA 02139; cw@space.mit.edu,jdr@space.mit.edu.

4 Also at Center for Space Science and Applied Research, Chinese Academy of Sciences, P.O. Box 8701, Beijing 100080, China.

5 Institute for Physical Science and Technology, University of Maryland, College Park, MD 20742; fm27@umail.umd.edu.

6 California Institute of Technology, Division of Physics, Mathematics and Astronomy, Pasadena, CA; ecs@srl.caltech.edu.
}

speed increased. A second shock, observed by $V 2$ at 2004.32 , was related to the October 29-30 events at $1 \mathrm{AU}$. The shock and MIR formed as a result of changes in the speed at $1 \mathrm{AU}$. These observations are discussed in $\S 3$. Section 3.3 shows that changes in the cosmic-ray intensity (CRI) were related to the quasi-periodic magnetic field variations at $V 2$ from before 2002.7 to $\approx 2003.37$, but there was no significant net change in the CRI. Both the large values of $B$ behind the shock and those in the MIR caused a steplike decrease in the CRI. The radial evolution of $V(t)$ and $B(t)$ in the flows out to $95 \mathrm{AU}$, determined from an MHD model, is discussed in $\S 4$.

The V2 plasma data used in the study are from the PLS experiment (Bridge et al. 1977) (PI: J. Richardson). The V2 magnetic field data are from the MAG experiment (Behannon et al. 1977) (PI: N. Ness). The $V 2$ cosmic-ray data are from the CRS experiment (Stone et al. 1977) (PI: E. Stone). As input to the Wang model, we use "level 2" hour averages of $B, V, N$, and the proton temperature $T$ measured by the MAG and SWEPAM instruments, respectively, on $A C E$ (PI: E. Stone).

\section{TRANSITION FROM QUASI-PERIODIC SPEED VARIATIONS TO FASTER APERIODIC FLOWS}

An abrupt transition to increasingly fast flows was observed by $V 2$ at $70.2 \mathrm{AU}$ at 2003.37 (line A in Fig. 1a), which was related to a similar transition observed by $A C E$ at $1 \mathrm{AU}$ at 2002.79 (A in Fig. $1 b$ ). The propagation time of the transition from $A C E$ to $V 2$ was $\approx 210$ days. The speed measured by $V 2$ (the solid dots in Fig. 1a) increased from an average of $\approx 390 \mathrm{~km} \mathrm{~s}^{-1}$ before the transition to $\approx 475 \mathrm{~km} \mathrm{~s}^{-1}$ at $\approx 2004.4$. A related increase in speed was observed by $A C E$ from $\approx 442 \mathrm{~km} \mathrm{~s}^{-1}$ near 2002.0 to $\approx 576 \mathrm{~km} \mathrm{~s}^{-1}$ near 2003.9 (Fig. $1 b$ ). Since $A C E$ and $V 2$ were not radially aligned during this period, the similar changes in speed must be related to relatively large-scale and/or persistent features in the solar wind between the Sun and 1 AU.

The abrupt transition to fast flows observed by $A C E$ at 2002.79 was related to the appearance coronal holes (Hundhausen 1977) crossing the solar equatorial plane on Carrington rotation (CR) 

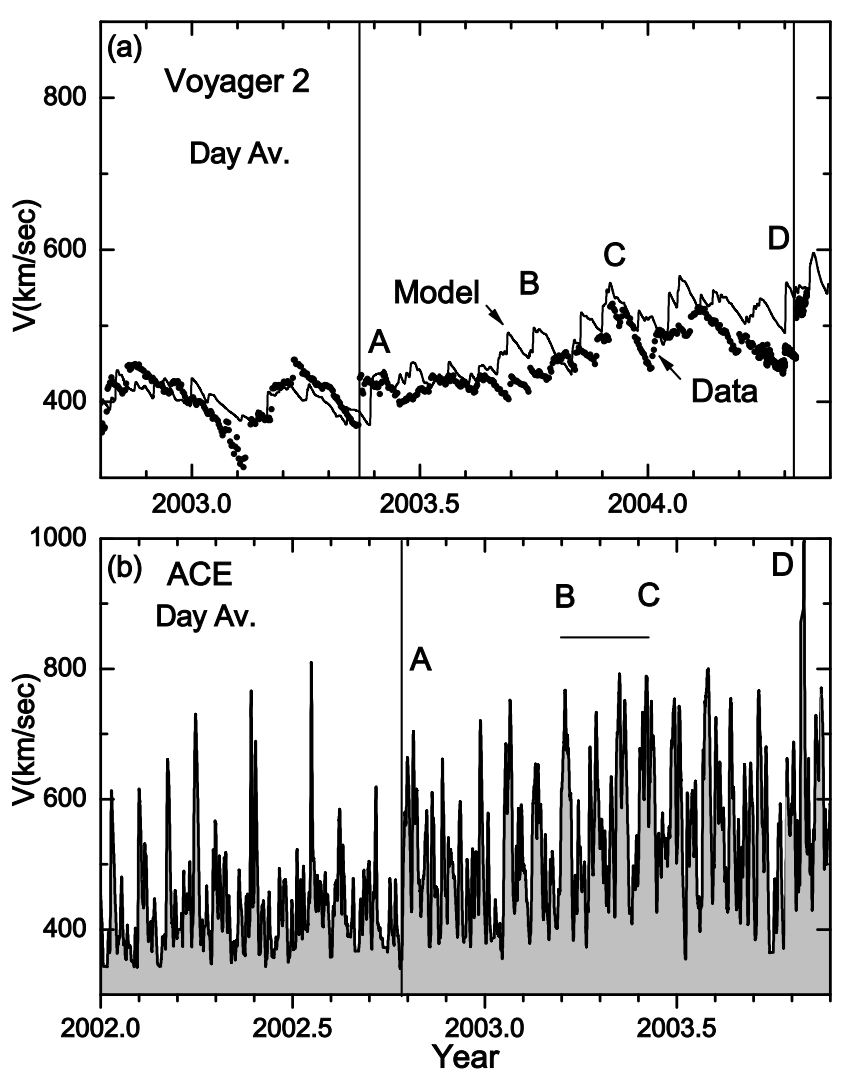

FIG. 1.-(a) Dots: daily averages of the solar wind speed measured by Voyager 2. The curve marked "Model" is the prediction of the model of Wang. (b) The daily averages of the speed measured by $A C E$ at $1 \mathrm{AU}$.

$\approx 1994 .^{7}$ Faster flows following $\approx 2003.19$ (B in Fig. $1 b$ ) were related to the appearance of more prominent coronal holes near the equatorial plane beginning $\approx C R \quad 1999$. A more detailed study is needed to establish the quantitative relation between the Sun and the streams observed by $A C E$, but that is beyond the scope of this paper.

We computed the speed profile at $V 2$ using a dynamical model for the evolution offlows in the solar wind, with the $A C E$ observations as boundary conditions. We used the numerical model of Wang \& Richardson (2001) and Wang et al. (2000), which is a deterministic, one-dimensional (spherically symmetric), multifluid, MHD model that includes pickup protons and the neutral interstellar gas (with a neutral hydrogen density equal to $0.09 \mathrm{~cm}^{-3}$ at the termination shock). Hourly averages of the $A C E$ magnetic field and plasma data measured at $1 \mathrm{AU}$ from 2002.0 to 2003.9 were used as input to Wang's model, and we calculated daily averages of $B(t), V(t), N(t)$, and $T(t)$ at the position of $V 2$. The speed at $V 2$ predicted by that model is shown by the curve marked "Model" in Figure $1 a$.

There is good agreement between both the trends and the basic features in the speed profile observed by $V 2$ and the predictions of Wang's model (Fig. 1a). First, two cycles of quasi-periodic variations in the speed discussed by Richardson et al. (2003) were observed by $V 2$ and predicted by the model from 2002.8 to $\approx 2003.37$. Second, the relatively large and very abrupt increase in speed observed by $V 2$ at $\approx 2003.37$ was predicted to occur at 2003.39. The observations show an increase from $\approx 367$ to $\approx 432 \mathrm{~km} \mathrm{~s}^{-1}$ across the speed jump, while the model predicts an increase in speed from $\approx 370$ to

\footnotetext{
7 See http://solar.sec.noaa.gov/ws/index.html.
}

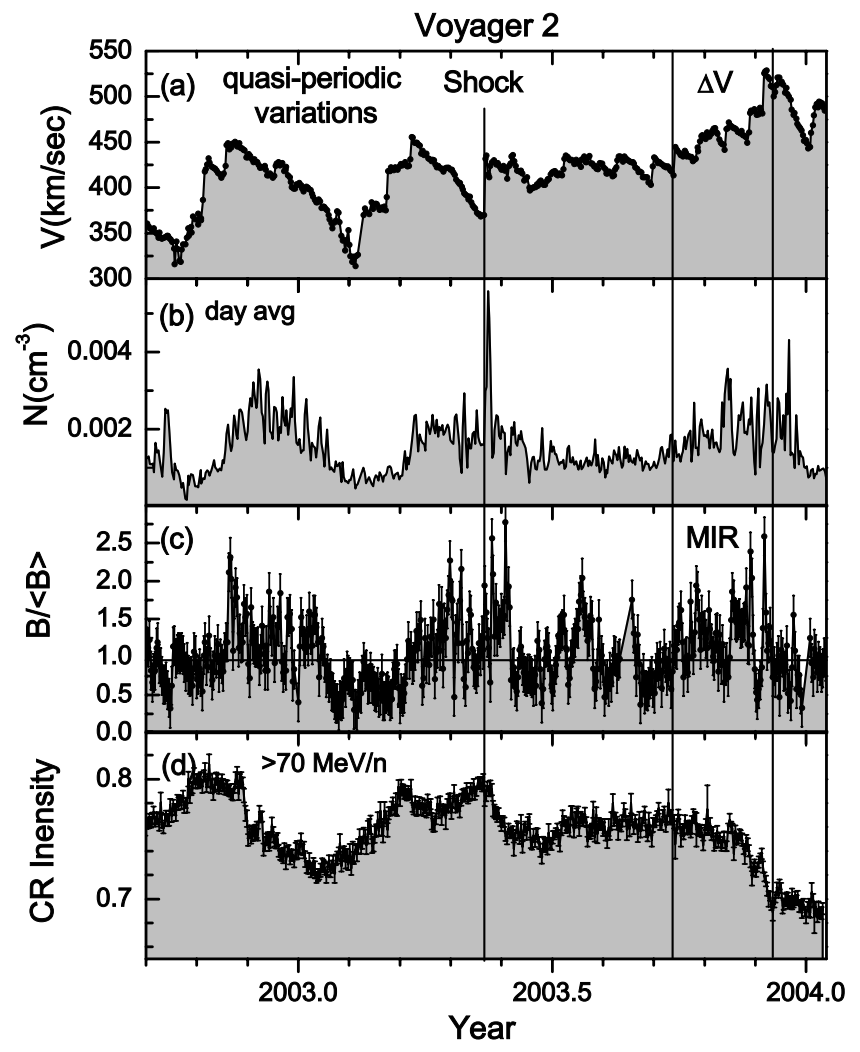

FIG. 2.-Daily averages of $(a)$ the solar wind speed, $(b)$ the solar wind density measured by Voyager 2, $(c)$ the magnetic field strength with $1 \sigma$ error bars, and $(d)$ the relative counting rate of cosmic rays more energetic than $70 \mathrm{MeV}$ nucleon $^{-1}$. Quasi-periodic correlated variations are observed from $\approx 2002.7$ to $\approx 2003.37$, after which there is an abrupt transition to a different state with increasing solar wind speeds.

$\approx 423 \mathrm{~km} \mathrm{~s}^{-1}$. The speed jump at $V 2$ was related to an increase in speed at $\approx 2002.79$ at $A C E$ (A in Fig. $1 b$ ). Third, an increase in $V$ in a series of steps from 2003.74 to 2003.94 from $\approx 417$ to $\approx 512 \mathrm{~km} \mathrm{~s}^{-1}$ was observed by $V 2$, and the model predicts a similar increase in speed (although different in detail). Finally, $V 2$ observed an abrupt increase in speed at 2004.32, and the model predicts a similar jump in speed at 2004.30 near 73.7 AU. This speed jump at $V 2$ was related to a very large increase in speed at $A C E$ at 2003.83 (D in Fig. $1 b$ ) that was associated with the October 29-30 events at 1 AU (Skoug et al. 2004). The relatively good agreement between the observed and predicted speed profiles, despite the generally large azimuthal and latitudinal differences in the positions of $V 2$ and $A C E$ implies that the basic structures observed in the speed profile had a latitudinal extent of at least $25^{\circ}$ and either had a large longitudinal extent or persisted for time scales longer than a solar rotation.

\section{THE SPEED, DENSITY, MAGNETIC FIELD STRENGTH, AND COSMIC RAYS AT VOYAGER 2}

\subsection{The Plasma and Magnetic Field Variations}

Daily averages of $V, N$, and $B /\langle B\rangle(\langle B\rangle=0.069 \mathrm{nT})$, and the CRI at $V 2$ from 2002.7 to 2004.04 are plotted in Figures $2 a, 2 b$, $2 c$, and $2 d$, respectively. At $\approx 2003.37, V 2$ observed an abrupt increase in $V$, a large spiky enhancement in $N$, and an abrupt increase in B. A small enhancement in the proton temperature, not shown in Figure 2, was also observed by $V 2$. Thus, the jump in $V$ at $\approx 2003.37$ discussed in $\S 2$, has the signature of a fast 
forward MHD shock. We cannot identify the structure as a true shock, because it occurred in a data gap, but we will call it a shock for brevity.

Prior to the shock, two cycles of the quasi-periodic variations discussed by Richardson et al. (2003) were observed by $V 2$. The speed, $N$, and $B /\langle B\rangle$ were positively correlated in these variations from before 2002.7 to 2003.37; the period of these oscillations was $\approx 120$ days. The simple pattern of correlated magnetic field and plasma variations at $V 2$ shown in Figure 2 was not observed by $A C E$ at $1 \mathrm{AU}$. However, one can see a quasi-periodic oscillation as a trend in the large-amplitude speed fluctuations at $A C E$. Section 4 shows that the quasi-periodic variations were produced by MHD processes beyond $1 \mathrm{AU}$. The quasi-periodic variations ended near the time of the shock at $V 2$.

Following the shock, there were three features in Figure 2 that are of special interest. First, immediately following the shock, there is a narrow region in which $B /\langle B\rangle, V$, and $N$ were relatively high. It is possible that the enhancements in $V, N$, and $B$ were produced by the advance of the shock into the sunward side of the last cycle of the quasi-periodic variations. Second, a speed gradient in which the speed increased stepwise from $\approx 417$ to $521 \mathrm{~km} \mathrm{~s}^{-1}$ during an interval of $\approx 2.8$ solar rotations was observed by $V 2$ from $\approx 2003.74$ to $\approx 2003.94$. This interval contains an MIR in which the magnetic field strength was relatively high (Burlaga 1995) that formed as a result of the overtaking and interaction of a series of streams.

\subsection{The Onset of a CMIR}

The unusual MIR at $V 2$ was being maintained by a speed gradient of $\approx\left(104 \mathrm{~km} \mathrm{~s}^{-1}\right) /(\approx 19.7 \mathrm{AU})$. Figure $2 a$ shows that the speed increased in a series of four jumps at $V 2$. These jumps occurred in the interval between the points $\mathrm{B}$ and $\mathrm{C}$ in Figure $1 a$, and they are associated with the arrival of four fast streams at 1 AU between points $\mathrm{B}$ and $\mathrm{C}$ in Figure $1 b$. Thus, the MIR at $V 2$ is related to the onset of a series of fast streams near the equatorial plane. The streams moved past $A C E$ at $1 \mathrm{AU}$ over the course of $\approx 3$ solar rotations ( $\approx 82$ days), beginning at 2003.19 .

The MIR observed by $V 2$ between $\approx 2003.74$ and $\approx 2003.94$ appears to be a CMIR whose sudden onset at $V 2$ is of special significance (CMIR refers to a corotating merged interaction region; Burlaga et al. 1993). The onset of such a CMIR could be produced near the solar equatorial plane either by the migration of coronal holes from high latitudes to the equatorial plane or by the sudden growth of near-equatorial coronal holes, as discussed by Burlaga et al. (2001) in regard to transitory corotating streams at $1 \mathrm{AU}$.

\subsection{Variations in the Cosmic-Ray Intensity}

Changes in the intensity of cosmic rays more energetic than $70 \mathrm{MeV}$ nucleon ${ }^{-1}$ were related to the magnetic field strength variations at $V 2$ from 2002.7 to 2004.04 (Figs. $2 c$ and $2 d$, respectively). The decreases in the CRI were associated with $B /\langle B\rangle>1$; the increases in the CRI were associated with $B /\langle B\rangle<1$; and the intervals with slowly varying CRI were associated with $B /\langle B\rangle$ fluctuating about $\approx 1$. This relationship between changes in the CRI and $B /\langle B\rangle$ is the CR- $B$ relation (Burlaga et al. 1985) that has been observed from 11 to $85 \mathrm{AU}$ (Burlaga et al. 1993, 2003a).

The quasi-periodic variations in $B /\langle B\rangle$ from 2002.7 to $\approx 2003.37$ caused variations in CRI, but they produced no appreciable net decrease in the CRI (Figs. $2 c$ and $2 d$ ). This is consistent with the pattern that one expects for the magnetic fields and speeds related to stationary corotating flows (Burlaga et al. 1993).

The enhancement in $B /\langle B\rangle$ following the shock at 2003.37 produced a small step decrease in the CRI. The CRI did not recover to its preshock value, suggesting that the enhancement in $B$ extended over a relatively wide range of longitudes, forming a barrier to cosmic rays. Following the postshock enhancement in $B, B /\langle B\rangle$ oscillated about unity until $\approx 2003.74$, during which time the CRI was approximately constant.

The passage of strong magnetic fields in the CMIR between $\approx 2003.74$ and $\approx 2003.94$ (Figs. $1 c$ and $2 d$ ) produced a relatively large steplike decrease in the CRI at V2. It is significant that this steplike decrease was associated with the arrival of fast recurrent streams at $1 \mathrm{AU}$ (Fig. 1b), unlike the steplike decreases associated with systems of transient flows at $1 \mathrm{AU}$ (Burlaga et al. 1984) and the corresponding shell-like global MIRs (GMIRs) at larger distances (Burlaga et al. 1993). Normally the CRI increases following the passage of a CMIR associated with a stationary corotating flow. In this case, however, the onset of the fast recurrent flows at $1 \mathrm{AU}$ and beyond was abrupt, hence nonstationary, producing the onset of a CMIR at $V 2$ that caused a step decrease in the CRI. It would be of interest to model this type of step decrease.

\section{RADIAL VARIATIONS OF THE SPEED AND MAGNETIC FIELD STRENGTH}

\subsection{Introduction}

The radial evolution of the speed profiles and the magnetic field strength profiles from 1 to 95 AU was computed with the model of Wang (Wang \& Richardson 2001; Wang et al. 2000). Hourly averages of $B, V, N$, and $T$ measured by $A C E$ at $1 \mathrm{AU}$ from 2002.0 to 2003.9 were used as input to the model. The profiles of $B(t)$ and $V(t)$ were computed at each of the distances $5,10,15, \ldots, 95 \mathrm{AU}$. In the following we discuss the radial evolution of the profiles at intervals of $10 \mathrm{AU}$ at distances from 15 to $95 \mathrm{AU}$. Recall that $A C E$ and $V 2$ were not radially aligned in general and were separated in latitude, so that one cannot expect precise agreement between the observations and predictions. However, one can expect reasonable agreement for features that are relatively large or persist longer than a solar rotation, or both.

\subsection{Radial Variation of the Speed Profile}

The computed radial evolution of the speed profiles is shown in Figure 3. The general increase in the speeds that was discussed in reference to Figure 1 in $\S 2$ is predicted at all distances from 15 to $95 \mathrm{AU}$, although the average $V$ decreased with increasing distance. The lines labeled A, B, C, and D correspond to the features identified by these letters in Figure $1 a$ and Figure $1 b$ for $A C E$ and $V 2$ data, respectively. The abrupt increase in speed at $1 \mathrm{AU}$ (A in Fig. 1b) evolved into a shocklike jump in speed that propagates at least to $95 \mathrm{AU}$, as indicated by line A in Figure 3.

Prior to the speed jump marked by the line A in Figure 3, one can see two cycles of the quasi-periodic speed variations forming between $\approx 15$ and $\approx 25$ AU. The first cycle and the beginning of the second cycle extended out to $95 \mathrm{AU}$.

A large radial gradient in speed was predicted between B and $\mathrm{C}$ in Figure 3 at $15 \mathrm{AU}$. This increase in speed is related to the fast streams between $\mathrm{B}$ and $\mathrm{C}$ in Figure $1 b$ at 1 AU. Figure 3 shows that the speed gradient persisted out to $65 \mathrm{AU}$, and Figure $1 a$ shows that it was observed by $V 2$ near $72 \mathrm{AU}$. We 


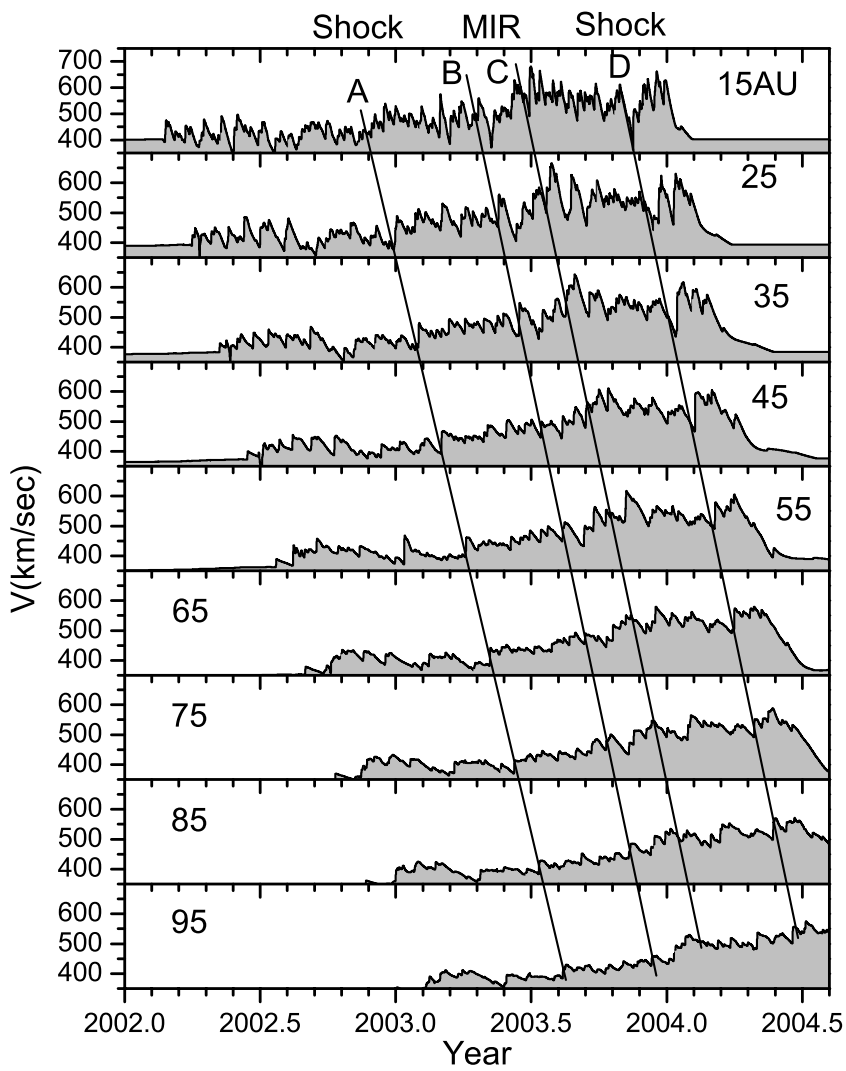

Fig. 3.-Radial evolution of the speed profiles predicted by the model. Lines A and D show the radial evolution of shocklike structures. The interval between lines $\mathrm{B}$ and $\mathrm{C}$ contains gradients in the speed that are related to the radial evolution of the MIR shown in Fig. 4.

have already discussed in $\S 2$ the relatively good agreement between the prediction and the $V 2$ observations of $V(t)$.

Finally, the model predicts a large increase in speed at $15 \mathrm{AU}$ beginning $\approx 2003.87$, the line marked $D$ in Figure 3, caused by a larger increase in speed at $1 \mathrm{AU}$ (D in Fig. 1b). This speed gradient evolved to a shocklike jump in speed that formed near $35 \mathrm{AU}$ (Fig. 3) and persisted to $95 \mathrm{AU}$.

\subsection{Radial Variation of the Magnetic Field Strength Profile}

The computed radial evolution of the magnetic field strength profiles is shown in Figure 4. An increase in the magnetic field strength, associated with the jump in speed at point A in Figure $1 b$ and line A in Figure 3, was predicted to propagate from 15 to $95 \mathrm{AU}$ (line A in Fig. 3). The increase in $B$ was associated with a shock at $V 2$ (point A in Fig. $1 a$ ).

Prior to the jump in $B$ marked by the line A in Figure 4, one can see the development of two cycles of the quasi-periodic magnetic field strength variations between $\approx 15$ and $\approx 55 \mathrm{AU}$. Each cycle formed by the merging of a series of enhancements in $B$ that is evident at $15 \mathrm{AU}$ in Figure 3.

The formation of an MIR can be seen between the lines $\mathrm{B}$ and $\mathrm{C}$ in Figure 4. At least two enhancements in $B$ at $15 \mathrm{AU}$ coalesced to an MIR at $\approx 35 \mathrm{AU}$. The evolution of this MIR was related to the evolution of the radial gradient in speed that was observed between lines $\mathrm{B}$ and $\mathrm{C}$ in Figure 3. The compression of the field in the MIR is driven by the speed gradient.

The predicted MIR corresponds to that observed by $V 2$ from $\approx 2003.74$ and $\approx 2003.94$ AU. Recall that the model predicted this MIR at $V 2$ using data from $A C E$, which is separated from

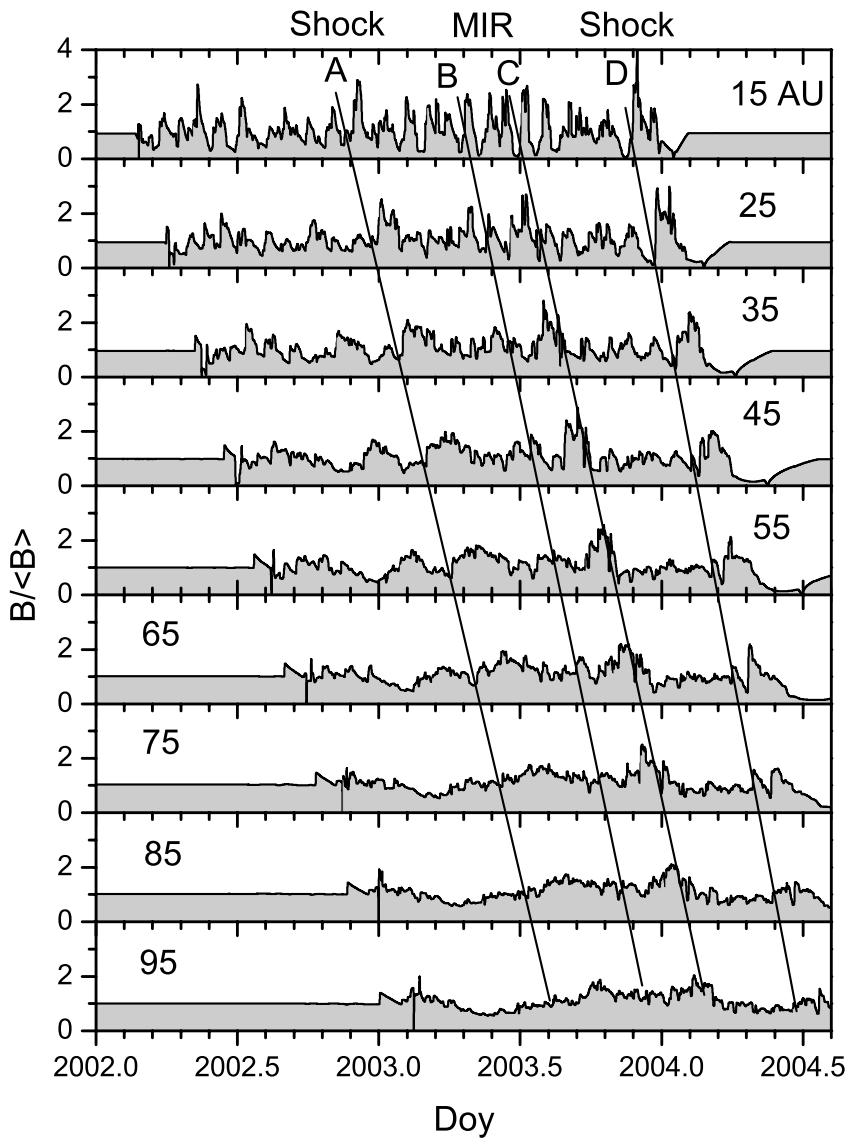

FIG. 4.-Radial evolution of the magnetic field strength profiles predicted by the model. Lines A and D show the radial evolution of shocklike structures. The interval between lines B and C contains an MIR related to the radial speed gradients between B and C in Fig. 3 .

$V 2$ in latitude and longitude. $V 2$ was at $288^{\circ}$ solar ecliptic longitude, while $A C E$ was between $169^{\circ}$ and $249^{\circ}$ during the interval from $\mathrm{B}$ to $\mathrm{C}$. Thus, the longitude difference started at $119^{\circ}$ and decreased to only $39^{\circ}$ during the interval from B to C. As a result, it is not surprising that the latter two streams at $1 \mathrm{AU}$, in particular, were also present at $V 2$. Perhaps the more relevant factor is that the high-speed stream was present for three solar rotations, and therefore most likely present for the entire time as the Sun rotated. The model indicates that during the last two rotations an MIR formed at $A C E$ 's longitude. Thus, it is reasonable to think that a ring of strong magnetic fields, extending around the Sun and to at least $25^{\circ}$ south latitude was formed. This MIR was formed by the merging of interaction regions associated with the onset of fast recurrent streams near the equatorial plane at $1 \mathrm{AU}$.

Finally, the model predicts a large increase in the magnetic field strength at $15 \mathrm{AU}$ beginning at $\approx 2004.87$, the line marked $D$ in Figure 4, which is associated with the speed increase at the line marked D in Figure 3. This is consistent with the formation of a shocklike structure that propagated out to $95 \mathrm{AU}$. The increase in $B$ was related to the relatively strong magnetic fields observed at $1 \mathrm{AU}$ in association with the fast flows on 2003 October 29-30 (Skoug et al. 2004). The amplitude of the jump in $B$ diminished with increasing distance from the Sun.

\section{SUMMARY AND DISCUSSION}

The change in the structure of the solar wind related to the recent transition from the maximum phase of solar activity to 
the declining phase was not a smooth transition. Rather, it was related to a sequence of features that we analyze in this paper. We show that the features observed by Voyager 2 can be understood within the framework of a time-dependent multifluid MHD model as a consequence of changes in the structure of the solar wind at $1 \mathrm{AU}$ related to the development of coronal holes.

A shocklike structure ("shock A") across which $V, N, T$, and $B$ observed by $V 2$ increased at 2003.37 marks the onset of the transition from relatively slow quasi-periodic flows to increasingly fast flows. The model predicts a shocklike transition at $V 2$ at 2003.39, related to an increase in speed observed by $A C E$ at $1 \mathrm{AU}$ at 2002.79. The shock moved through the heliosphere from 15 to $95 \mathrm{AU}$. The speed increase at $A C E$ at $1 \mathrm{AU}$ was related to the appearance of coronal holes crossing the solar equatorial plane on Carrington rotation $(C R) \approx 1994$.

Two cycles of quasi-periodic variations in $V$ and $B$ were observed by $V 2$ prior to the shock, from 2002.8 to $\approx 2003.37$. The model predicts variations in $V$ and $B$ that agree with those observed by $V 2$. The quasi-periodic variations formed between 1 and $\approx 25$ AU. Each cycle formed by the merging of a series of enhancements in $V$ and $B$.

A corotating merged interaction region (CMIR) driven by a radial gradient in $V$ (in which $V$ increased from $\approx 417$ to $521 \mathrm{~km}$ $\mathrm{s}^{-1}$ in a series of jumps over an interval of $\approx 2.8$ solar rotations) was observed by $V 2$ from 2003.74 to 2003.94 . The model predicts a similar radial gradient in $V$ in a series of steps at $V 2$. This CMIR and speed gradient probably formed as a result of the overtaking and interaction of roughly four fast recurrent streams that moved past $A C E$ over the course of $\approx 3$ solar rotations beginning 2003.19. The onset of the CMIR caused a step decrease in the cosmic-ray intensity. The CMIR might have had the form of a ring of strong magnetic fields, extending around the Sun and to at least $25^{\circ}$ south in latitude. The onset of the
CMIR observed by $V 2$ was related to a relatively abrupt onset of coronal holes near the equatorial plane. Such a CMIR could be produced near the equatorial plane either by the migration of coronal holes from high latitudes or by the sudden growth of near-equatorial coronal holes.

A second shocklike increase in speed was observed by $V 2$ at 2004.32. The model predicts that a shocklike increase in $V$ and $B$ formed near $\approx 35 \mathrm{AU}$, evolving from a very large increase in speed observed by $A C E$ at 2003.83 . These increases in $V$ and $B$ are related to the fast transient flows and relatively strong magnetic fields observed at 1 AU on 2003 October 29-30.

Changes in the intensity of cosmic rays more energetic than $70 \mathrm{MeV}$ nucleon $^{-1}$ were related to the magnetic field strength variations at $V 2$ from 2002.7 to 2004.04. The quasiperiodic variations in $B /\langle B\rangle$ observed from 2002.7 to 2003.37 produced variations in CRI, but they produced no appreciable net decrease in the CRI, as expected for stationary corotating flows. The enhancement in $B /\langle B\rangle$ following the shock was related to a small step decrease in the CRI. The CMIR produced a second, larger steplike decrease in the CRI at $V 2$.

N. F. Ness was partially supported by JPL Contract 959167. J. D. Richardson and C. Wang were partially supported by NASA under JPL contract 959203 to MIT and NASA grant NAG511623. C. Wang was also supported in part by NNSFC 40204009 from China. We thank S. Kramer and T. McClanahan for support in processing the magnetic field data, and M. Acuña for his continued assistance in evaluating the measurements. The $A C E$ plasma and magnetic field data used in this work were obtained from the $A C E$ Science Center. The $A C E$ magnetic field data were provided by $\mathrm{C}$. Smith and N. Ness. The $A C E$ plasma data were provided by R. Skoug and D. McComas.
Behannon, K. W., Acuna, M. H., Burlaga, L. F., Lepping, R. P., Ness, N. F., \& Neubauer, F. M. 1977, Space Sci. Rev., 21, 235

Bridge, H. S., Belcher, J. W., Butler, R. J., Lazarus, A. J., Mavretic, A. M., Sullivan, J. D., Siscoe, G. L., \& Vasyliunas, V. M. 1977, Space Sci. Rev., 21, 259

Burlaga, L. F. 1995, Interplanetary Magnetohydrodynamics (Oxford: Oxford Univ. Press)

Burlaga, L. F., Goldstein, M. L., McDonald, F. B., \& Lazarus, A. J. 1985, J. Geophys. Res., 90, 12027

Burlaga, L. F., Harvey, K. L., \& Sheeley, N. R. 2001, J. Geophys. Res., 106, 24915

Burlaga, L. F., McDonald, F. B., \& Ness, N. F. 1993, J. Geophys. Res., 98, 1

Burlaga, L. F., McDonald, F. B., Ness, N. F., Schwenn, R., Lazarus, A. J., \& Mariani, F. 1984, J. Geophys. Res., 89, 6579

Burlaga, L. F., Ness, N. F., McDonald, F. B., Richardson, J. D., \& Wang, C. 2003a, ApJ, 582, 540

\section{EFERENCES}

Burlaga, L., Ness, N., Stone, E., McDonald, F., Acuna, M. H., Lepping, R., \& Connerney, J. 2003b, Geophys. Res. Lett., 30, 2072

Hundhausen, A. J. 1977, in Coronal Holes and High Speed Wind Streams, ed.

J. B. Zirker (Boulder: Colorado Assoc. Univ. Press), 225

Krimigis, S. M., et al. 2003, Nature, 426, 45

McDonald, F. B., Stone, E. C., Cummings, A. C., Heikkila, B., Lal, N., \& Webber, W. R. 2003, Nature, 426, 48

Richardson, J. D., Wang, C., \& Burlaga, L. F. 2003, Geophys. Res. Lett., 30, 2207

. 2004, Adv. Space Res., 34, 150

Skoug, R. M., Gosling, J. T., Steinberg, J. T., McComas, D., Smith, C. W., Ness, N., Hu, Q., \& Burlaga, L. 2004. J. Geophys. Res., 109, 09102

Stone, E. C., Vogt, R. E., McDonald, F. B., Teegarden, B. J., Trainor, J. H., Jokipii, J. R., \& Webber, W. R. 1977, Space Sci. Rev., 21, 355

Wang, C., \& Richardson, J. D. 2001, J. Geophys. Res., 106, 29401

Wang, C., Richardson, J. D., \& Gosling, J. T. 2000, J. Geophys. Res., 105, 2337 